\title{
Detection of pulsed blood flow through a molar pulp chamber and surrounding tissue in vitro
}

\author{
S. Knörzer, ${ }^{1,2}$ K.-A. Hiller, ${ }^{1}$ M. Brandt, ${ }^{3}$ A. Niklas, ${ }^{4}$ J. Putzger, ${ }^{5}$ G. J. Monkman, ${ }^{6}$ \\ S. N. Danilov, ${ }^{5}$ S. Ganichev ${ }^{5}$ I. Schulz, ${ }^{6}$ G. Schmalz ${ }^{1,7 *}$ \\ ${ }^{1}$ Department of Conservative Dentistry and Periodontology, University Medical Center Regensburg, University \\ of Regensburg, Regensburg, Germany \\ ${ }^{2}$ Private practice, Amberg, Germany \\ ${ }^{3}$ Department of Orthodontics, University Hospital of RTWH Aachen, Germany \\ ${ }^{4}$ Private practice, Kulmbach, Germany \\ ${ }^{5}$ Department of Physics, University of Regensburg, Germany \\ ${ }^{6}$ Mechatronics Research Unit, University of Applied Sciences Regensburg, Germany \\ ${ }^{7}$ Department of Periodontology, University of Bern, Switzerland
}

${ }^{*}$ Corresponding author:

Gottfried Schmalz

E-mail: gottfried.schmalz@ukr.de

\begin{abstract}
Objectives: Due to severe limitations of dental pulp sensitivity tests, the direct recording of pulsed blood flow, using photoplethysmography (PPG), has been proposed. In vivo evaluation is methodologically difficult and in vitro models have hitherto been adversely influenced by shortcomings in emulating the in vivo situation.

Consequently, the aim of this study was to test an improved data acquisition system and to use this configuration for recording pulsed blood in a new model.

Materials and Methods: We introduced a PPG signal detection system by recording signals under different blood flow conditions at two wavelengths (625 and $940 \mathrm{~nm}$ ). Pulsed blood flow signals were measured using an in vitro model, containing a molar with a glass pulp and a resin socket, which closely resembled in vivo conditions with regard to volumetric blood flow, pulp anatomy and surrounding tissue.

Results: The detection system showed improved signal strength without stronger blanketing of noise. On the tooth surface, it was possible to detect signals emanating from pulsed blood flow from the glass pulp and from surrounding tissue at $625 \mathrm{~nm}$. At $940 \mathrm{~nm}$, pulp derived signals were recorded, without interference signals from surrounding tissue.

Conclusion: The PPG-based method has the potential to detect pulsed blood flow in small volumes in the pulp and (at $625 \mathrm{~nm}$ ) also in adjacent tissues.

Clinical Relevance: The results show the need for clear differentiation of the spatial origins of blood flow signals of any vitality test method to be applied to teeth.
\end{abstract}

Keywords: dental pulp tests, laser Doppler flowmetry, photoplethysmography, pulpal blood flow, pulp vitality

\author{
Acknowledgments \\ Support by Deutsche Forschungsgemeinschaft projects (SCHM 386/3, GA-501/10 and MO 2196/1), \\ Bundesministerium für Bildung und Forschung (BMBF) and Anwendungszentrum "Miniaturisierte Sensorik" \\ funded by the Bavarian Government is gratefully acknowledged. We declare to have no relevant financial \\ interests in this manuscript.
}




\section{Introduction}

Clinically available test methods for the evaluation of dental pulpal tissue vitality suffer from severe limitations. The nerve response (sensitivity) toward commonly used thermal or electric stimuli does not necessarily correspond to pulpal blood flow [1, 2], which is generally regarded as a main criterion for tissue vitality. This is especially true, following trauma [1], hypoxia [3, 4] or for teeth with incomplete root formation [4]. In a systematic review [2], it has been highlighted that scientific evidence for the accuracy of assessing pulp vitality by current sensitivity test methods is insufficient.

As alternatives, different objective, non-invasive methods for the direct assessment of pulpal blood flow have been proposed and evaluated during recent decades.

Photoplethysmography (PPG) employs light, e.g. emitted by a light emitting diode (LED), whose transmission through the tooth is dependent on blood flow. The output signal is then detected by a photosensor on the opposite side of the tooth [5, 6] or in reflection [7]. Pulse oximetry (PO) is based upon the same technical principle as PPG, but uses two wavelengths (red and infrared), allowing the determination of haemoglobin oxygen saturation [8-10]. Laser Doppler flowmetry (LDF) uses the Doppler frequency shift of laser light by reflection from moving erythrocytes to detect blood flow. This technique has been established as the gold standard of experimental blood flow measurements in soft tissues [11, 12]. Laser speckle imaging (LSI) $[13,14]$ or ultrasonic flowmetry (UFM) $[15,16]$ have also been examined on teeth in vitro [14] and in vivo [13, 15, 16] within pilot studies.

The problems associated with each of these methods have been described elsewhere [17]. In general, they suffer from the problem that the signals detected from a tooth may not only result from blood flow in the dental pulp itself, but also from blood flow through tissue surrounding the tooth. This can be observed from in vivo studies reporting on blood flow signals recorded with PPG $[6,18]$, PO [19, 20] or LDF [21-26], even when applied to non- 
vital or root-filled teeth. This risk of recording blood flow in other than pulpal tissue has also been discussed for UFM [15].

One potential source of such interference signals is dental hard tissues, which act as light guides and can scatter rays which are directed onto the crown surface, through the root into surrounding structures. This is particularly apparent in LDF [27, 28]. Thus, recorded signals may derive from blood flow in the periodontal ligament, the surrounding bone or from the gingiva. Using the PPG method, Fein et al. [6] placed an opaque plastic strip between the tooth and the marginal gingiva, which reduced the recorded signal amplitude only slightly, while local anesthesia with the vasoconstrictor epinephrine (1:50.000) applied to the gingiva caused a drastic reduction in the recorded signal. This indicates a substantial influence from gingival blood flow on PPG signals detected through teeth. In an attempt to avoid or reduce interfering PPG signals, different dental dams and foils were examined $[6,17,18]$, revealing inconsistent results.

Besides this uncertainty on the spatial origin of detected signals, another problem with the development of objective pulp vitality test methods is the lack of a gold standard, with which in vivo data from new test methods can be compared [2, 6]. Here, in vitro models which closely replicate the in vivo situation are advantageous, because the different parameters (e.g. signal sources) can be defined and no control test methods are required [2931].

In a recent in vitro study from our research group [17], we found that pulsed blood flow could be detected in a tooth-gingiva-model, using the PPG technique. Serving as a proof-ofprinciple, this model had severe limitations: The conditions for blood flow rates in this model (23.5 ml/min) greatly exceeded physiological pulpal (20 - $60 \mathrm{ml} / \mathrm{min}$ per $100 \mathrm{~g}$ tissue [4, 32, 33]) and gingival (30 ml/min per $100 \mathrm{~g}$ tissue [34]) in vivo blood flow rates. Furthermore, the use of a straight glass capillary or silicone tube in the molar to emulate the pulpal blood flow in the tooth-gingiva-model did not resemble in vivo conditions in either shape or size. 
As a consequence, the hypotheses for the present study were: (1) pulsed blood flow signals can be detected with an improved PPG recording device, whereas constant, nonpulsed blood flow or static blood reveals no periodically modulated signal; and (2) signals from both pulpal and gingival blood flow can be detected, either separately or combined, in a new in vitro model emulating anatomical in vivo conditions more closely than in the former study. Therefore, the aim of this work was to evaluate an improved PPG-based signal detection system with an established in vitro tooth-gingiva-model [17] and to use this system to detect signals resulting from pulsed blood flow in a new in vitro model with dimensions of a pulp chamber close to the in vivo situation whilst emulating the in vivo surrounding tissues. 


\section{Materials and methods}

\section{Blood preparation}

Human leukodepleted erythrocyte concentrates (EC; Blood bank, University Hospital Regensburg, Germany) were supplemented with a $10 \%$ copolymer surfactant/NaCl solution (Synperonic F68, CAS [9003-11-6]; Serva Electrophoresis, Heidelberg, Germany) in a ratio of 1:100 Synperonic solution/EC. Such blood preparations from four donors (EC 1-4) were used as described below.

\section{Light emitting and recording systems}

As in our previous studies [17, 35], we used light from two high power-LEDs with wavelengths of $625 \mathrm{~nm}$ (red) and $940 \mathrm{~nm}$ (infrared) with the lamp current $\left(\mathrm{I}_{\mathrm{L}}\right)$ adjusted to $0.1 \mathrm{~A}$. The recording system used in this study has been described in detail elsewhere by Schulz et al. [35]. Briefly, for the detection, conversion, filtering, amplification and light to voltage conversion, a monolithic photosensor (OPT101PG4; Burr-Brown, TI, Dallas, USA) in addition to specifically designed and custom built devices and laboratory software (LabView 2012, Version 12.0f3 (32bit); National Instruments, Austin, Texas, USA), were used. Parallel to the voltage signal, vascular pressure in both circuits was recorded using piezoresistive membrane pressure sensors (40PC015G1A, Honeywell, Morristown, NJ, USA).

\section{Flow-through models of a tooth with surrounding tissues}

For the initial part of the study, testing the suitability of the new signal recording system, we used the tooth-gingiva-model (TGM) developed by Niklas et al. [17]. Briefly, this model consisted of a human mandibular molar with a straight glass capillary (inner diameter: $1.0 \mathrm{~mm}$ ) placed through the crown, from the central fissure to the bifurcation. The roots of the molar were embedded in an acrylic resin socket (Paladur clear; Heraeus Kulzer, Hanau, 
Germany), which contained two canals (inner diameter: ca. $3 \mathrm{~mm}$ ) surrounding the roots, by which gingival blood flow was emulated. The capillary and the canals were connected separately to peristaltic pumps, creating a pulsed pulpal and gingival blood flow of $23.5 \mathrm{ml} / \mathrm{min}$ (all flow rates measured by weight) at a pulse frequency of $1 \mathrm{~Hz}$, resulting in a mean vascular pressure in the pulpal and the gingival cycle of $43 \mathrm{mmHg}$ and $17 \mathrm{mmHg}$, respectively. The experimental configuration is shown in Fig. 1a.

A constant, non-pulsed blood flow was achieved by using two 50 ml-burettes (Silberbrand; Brand, Wertheim, Germany) in the apparatus (Fig. 1b). This resulted in $25 \mathrm{mmHg}$ vascular pressure and $15.5 \mathrm{ml} / \mathrm{min}$ volumetric flow for the pulpal and 10 $15 \mathrm{mmHg}$ and $15.0 \mathrm{ml} / \mathrm{min}$ for the gingival blood flow. The static pressure of the erythrocyte concentrate in the burettes was used to generate the pulpal and gingival blood flow, while in this configuration the pumps were only used to maintain the burette filling level constant and prevent a decline in static pressure.

For the second part of the study, we developed a new model (Fig. 2). This glass pulp model comprised an intact human mandibular molar, which was placed into an anatomically formed socket, emulating the tissues surrounding the tooth. The roots were enlarged retrogradely with endodontic K-files (K-files; VDW GmbH, Munich, Germany) to ISO size 100 and the tooth cut into two halves. A hollow glass form (Duran borosilicate glass; Duran, Wertheim, Germany) was then fitted into the pulp chamber and the root canals using a heatproof cast, allowing the blood preparation to enter into one root (inner diameter: ca. 0.1$0.15 \mathrm{~mm}$, measured with root canal files) before passing through the coronal part (inner diameter: ca. $0.5 \mathrm{~mm}$ ) and to exit through the other root (inner diameter: ca. 0.1-0.15 mm) (Fig. 3).

The socket of the model consisted of an inner part emulating the surrounding bone and an outer part emulating the gingiva. For the inner part an acrylic resin mixture (PALA Paladur; Heraeus Kulzer, Hanau, Germany, 0.36 g transparent powder +1.14 g pink powder + 
$1.1 \mathrm{ml}$ liquid) was used. This mixture has similar light transmission properties to those of a whole porcine jaw at red to infrared wavelengths [36]. For the outer part, a soft relining silicone (Mollosil plus; Detax, Ettlingen, Germany) was used, into which a flexible silicone tube (Tygon R3607; inner diameter: 0.25 mm; Saint-Gobain Performance Plastics, Paris, France) was embedded at the position of the marginal gingiva to emulate gingival blood flow (Fig. 2). Finally, the two tooth halves with the glass insert were tightly and precisely secured together and placed into the socket without the use of any resin or bonding agents.

The flow rate within the glass pulp model was adjusted to $0.35 \mathrm{ml} / \mathrm{min}$ with a pulse frequency of $1.2 \mathrm{~Hz}$. The vascular pressure in the glass pulp was $450-600 \mathrm{mmHg}$ with an amplitude of ca. $100 \mathrm{mmHg}$. In the gingival part, it was 350 - $550 \mathrm{mmHg}$ with an amplitude of ca. $200 \mathrm{mmHg}$.

\section{Experimental}

We performed five experimental series, using different erythrocyte concentrates (EC): In the initial parts of the study (hypothesis 1), pulsed (EC 1) and non-pulsed blood flow (EC 2) were recorded in the tooth-gingiva-model [17] for 10 seconds. In a third series, signals were recorded with pulsed blood flow for 10 seconds, which was then ceased and signals measured continuously for a further 10 seconds (EC 1). In the second part of the study (hypothesis 2), pulsed blood flow was recorded using the glass pulp model. This experiment was performed twice, with ECs from two different donors (EC 3 and 4). Measurements with pump cessation were also taken with the new glass pulp model (EC 4).

As in the previous study by Niklas et al. [17], recordings were made for two wavelengths (625 nm and $940 \mathrm{~nm}$ ) and three flow modes: blood flowing only through the tooth (T), only through the gingiva (G) and both combined (TG), resulting in six experiments for each experimental series. 


\section{Data treatment}

The readout signals from the recording system were displayed on a monitor screen showing curves of the recorded voltage (Fig. 4) and pressure signals against time. Pressure signals underwent no further quantitative analysis. Periodic, pulse synchronous, repetitive signal structures (modulation) of the voltage signal were identified as the example in Fig. 4 shows. For quantitative analysis, the amplitude of the voltage signal modulation ( $\Delta U$ in Volt) was used (Fig. 4): The first three seconds of the blood flow recording were omitted. Finally, the following modulation amplitudes $\left(\Delta \mathrm{U}_{1}, \Delta \mathrm{U}_{2}\right.$ and $\left.\Delta \mathrm{U}_{3}\right)$ were calculated as the difference between the respective maxima and minima of each of the three modulation amplitudes, from which the median was then used as the representative value for these experiments. Each experiment was performed five times. From the representative values from these five experiments, a median with $25 \%$ - and 75 \%-quantiles were depicted.

For statistical analysis, pairwise non-parametric tests (Mann-Whitney-U-Test) using the SPSS software (SPSS 22.0; IBM, Ehningen, Germany) was applied with a significance level of $\alpha=0.05$. 


\section{Results}

Results of the first part of the study (hypothesis 1), testing the new signal recording system using the tooth-gingiva-model and pulsed blood flow (T, G, TG), are shown in Fig. 5.

For all experiments, periodic, pulse synchronous signal modulations could be detected and $\Delta \mathrm{U}$ values derived (example for TG at $625 \mathrm{~nm}$ in Fig. 4). For $625 \mathrm{~nm}$, the $\Delta \mathrm{U}$ value for the blood flow in the gingiva $(G)(0.1355 \mathrm{~V})$ was significantly lower than those of the tooth (T) $(0.9065 \mathrm{~V})$ and both tooth and gingiva $(\mathrm{TG})(0.9918 \mathrm{~V})(\mathrm{p} \leq 0.008)$. For $940 \mathrm{~nm}$, the $\Delta \mathrm{U}$ values of TG (5.3899 V), G (3.6542 V) and T (2.3501 V) showed a decline in that order. With the exception of T vs. TG at $625 \mathrm{~nm}$ (marked with * in Fig. 5), all $\Delta \mathrm{U}$ values were pairwise significantly different ( $\mathrm{p} \leq 0.008$ in all cases).

The signal voltage curves from the measurements using a constant, non-pulsed blood flow in the tooth-gingiva-model showed an unstructured shape with no periodic modulation related to the blood pulse for any wavelength (625 nm and $940 \mathrm{~nm}$ ) or flow mode (T, G and TG). Examples from each experiment are shown in Fig. 6. No quantitative analysis of the $\Delta \mathrm{U}$ values was possible. The pressure signals showed constant pressure.

The measurements with pump cessation and the tooth-gingiva-model showed synchronously pulse modulating voltage signals, which changed into aperiodic baseline signals following cessation of the pulsed blood flow (Fig. 7). The same applied to the pressure signals (example pressure graphs for TG at $625 \mathrm{~nm}$ and $940 \mathrm{~nm}$ in Fig. 7). Again, no exact quantitative analysis of the $\Delta \mathrm{U}$ values was possible.

The results of the second part of the study (hypothesis 2) with the glass pulp model and pulsed blood flow are shown in Fig. 8. In all experiments, except for the recordings with blood flow only in the gingiva at $940 \mathrm{~nm}$, periodic, synchronous pulse modulation of the voltage signals could be detected. At $625 \mathrm{~nm}$, the amplitudes $(\Delta \mathrm{U})$ of the voltage signal for the circulation mode G (EC 3: $0.0517 \mathrm{~V}$; EC 4: $0.0247 \mathrm{~V}$ ) were pairwise significantly lower 
than for T (EC 3: 0.2202 V; EC 4: 0.4648 V) and TG (EC 3: 0.2292 V; EC 4: 0.4359 V) ( $\mathrm{p} \leq 0.008$ for both ECs and all cases). T and TG showed no significant differences in their $\Delta U$ values ( $p>0.05$ for both ECs). At $940 \mathrm{~nm}$, no modulation was detected for G. T (EC 3: 1.3829 V; EC 4: 2.6787 V) and TG (EC 3: 1.0484 V; EC 4: 1.9318 V) again showed no significant differences in their $\Delta U$ values ( $p>0.05$ for both ECs). Comparing the results between the two donors (EC 3 and 4), both ECs showed the same ranking for T, G and TG at $625 \mathrm{~nm}$ and $940 \mathrm{~nm}$. For T and TG, $\Delta \mathrm{U}$ values were significantly lower with EC 3 than with EC 4 at both wavelengths ( $p \leq 0.032$ ). Gingival blood flow $(G)$ was very small for both ECs. At $625 \mathrm{~nm}$ it was only just detectable but not detectable at $940 \mathrm{~nm}$.

The measurements with pump cessation and the glass pulp model showed results analogous to those obtained with the tooth-gingiva-model. No modulation was detected after cessation of pumping (Fig. 9). 


\section{Discussion}

\section{New recording device}

In the first part of the study, a new recording system was tested regarding different blood flow conditions (hypothesis 1). Contrary to the case already reported by Schulz et al. [35], we used a new optical fiber cable with a reinforced jacket but an unchanged fiber core, reduced lamp current $\left(\mathrm{I}_{\mathrm{L}}\right)$ and additionally tested this device for constant blood flow and zero flow conditions.

For the generation of a constant blood flow without pulsation, two burettes were used. Our detection system has been specifically designed to detect very small volumetric flow rate changes, expressed as voltage signal modulation, with a high temporal resolution (Fig. 4). For this reason, we preferred a non-mechanical flow generation as opposed to perfusion pumps [31, 37], in order to avoid spurious signals due to flow rate inconsistencies.

In accordance with data from Schulz et al. [35], the results from the first part of the study show that using the new signal detection system, a periodic synchronous pulsed blood flow signal can be detected in vitro at both wavelengths (625 and 940 nm, Fig. 5) and that the amplitudes of these derived PPG signals ( $\Delta U$ values) are larger than those derived using the apparatus from Niklas et al. [17] (Fig. 10) who used the same tooth gingiva model and circulation apparatus as we did in this study. He made identical measurements to ours but used an earlier version of a signal detection unit and used a 5 times higher incoming current for the LED.

Despite the signal enlargement, we observed no enlargement in the noise base in our measurements. Within our study these results primarily demonstrated the technical suitability of the new configuration, enabling us to reduce the blood flow in the second part of the study 
(blood flow generation at anatomical blood volumes). A further quantitative comparison of the measurement devices was not within the focus of our study.

Further to the results from Schulz et al. [35], it was shown that the system is not sensitive to constant blood flow or static blood. Possible extrinsic sources of signal interference such as ambient light [5, 9, 29, 38], electromagnetic radiation [9] or mechanical motion $[5,38]$ were maintained constant during the measurements, since they were not within the mandate of this study. Kahan et al. [38] discussed the risk of generating a pseudo signal from unspecific ambient noise through extensive signal filtering and amplification within a narrow range of frequencies. This could be excluded for our measurements as no modulated signals were detected in the absence of pulsed blood flow.

While the voltage signal reverted to a ground line for static blood (after cessation of pumping), the constant circulation lead to an unstructured signal which was not constant, indicating cell movements between light source and photosensor. These signal fluctuations could arise from transmission changes caused by local hematocrit inhomogeneities [31, 39], which have also been observed by Vongsavan and Matthews [40].

The signals shown are examples intended for comparison purposes. The main aim of the investigation was to detect clear signal structures from which $\Delta \mathrm{U}$-values could be derived. No exact measurement of the signal to noise ratio was made, however the filter suppression in the circuits used was at least $20 \mathrm{~dB}$ which was found to be adequate.

\section{Blood flow generation at anatomical blood volumes}

In the second part of the study, we aimed to emulate pulpal and gingival blood flow separately and more closely to in vitro conditions and to derive signals in a new in vitro model (hypothesis 2). By creating the glass pulp model, it was possible to avoid substantial losses of dental hard tissue from the model tooth, as in the case of drilling holes with 
diameters of up to several millimetres through the tooth crown and root [28, 30], and to emulate a blood supply through the apical foraminae and the dental roots. The inner diameter of the glass pulp roots of $100-150 \mu \mathrm{m}$ is equivalent to the size of the unbranched, centrally running arterial and venous vessels of the root pulp [32]. The coronal part of the model tooth pulp chamber had been enlarged just as much as necessary (ca. $0.5 \mathrm{~mm}$ ) to fit exactly in the glass pulp, whose inner volume can thus be assumed to be within in vivo scale of a crown pulp. Emulation of the ramified and anastomosed coronal vascular plexus remained difficult. However, with the varying diameters of the glass pulp, a non-laminar and partly turbulent blood flow was created to emulate multidirectional blood flow. For our model, we decided not to insert porous glass material into the glass pulp in order to avoid blood coagulation [41] and thus irreversible changes to the glass pulp during measurements.

We chose a hollow form of glass instead of directly filling erythrocyte concentrate into the tooth pulpal chamber $[29,37]$ to prevent erythrocytes from entering into the dentinal tubules, which could have caused irreversible changes to the optical properties of the tooth during measurements. To maintain these properties, neither liner nor bonding materials were used for sealing the tubules. The only interventions on the tooth were the vertical cut, the careful enlargement of the pulp chamber and rounding of sharp edges to enable the insertion of the glass pulp.

The tooth socket was formed anatomically and built from a resin, suitable for the emulation of the optical properties of a porcine jaw. These had been determined by transmission measurements on complete porcine mandibles and had been compared to different mixtures of dental resins [36] (Fig. 11). The resin mixture used in our study closely resembled these properties and for this reason was used as an approximation to in vivo conditions.

By the readjustment of the pumps, the use of smaller silicone tubes and glass tube connectors, the blood flow rate was reduced from $23.5 \mathrm{ml} / \mathrm{min}$ to $0.35 \mathrm{ml} / \mathrm{min}$ for the pulpal 
and the gingival cycle, which means a $98.5 \%$ decrease. With a volumetric in vivo blood flow rate of $20-60 \mathrm{ml} / \mathrm{min}$ per $100 \mathrm{~g}$ of pulpal tissue [4, 32, 33], an average in vivo pulp chamber volume $53 \mathrm{~mm}^{3}$ [42] and a presumed pulpal tissue density of $1 \mathrm{~g} / \mathrm{mm}^{3}$, an in vivo reference value for pulpal blood flow of $0.01-0.045 \mathrm{ml} / \mathrm{min}$ can be calculated for a first molar. This low value was not reached, but a good approach was possible. Concerning gingival blood flow, the volumetric blood flow in the gingiva of dogs is about $30 \mathrm{ml} / \mathrm{min}$ per $100 \mathrm{~g}$ tissue [34]. With an assumed tissue density of $1 \mathrm{~g} / \mathrm{mm}^{3}$, our volumetric gingival blood flow of $0.35 \mathrm{ml} / \mathrm{min}$ would be sufficient for the perfusion in a tissue volume of $1.2 \mathrm{~cm}^{3}$, which matches the arbitrary volume of the marginal gingiva used in our model socket.

Vascular pressures were 450 - $600 \mathrm{mmHg}$ for the pulp and 350 - $550 \mathrm{mmHg}$ for the gingiva, which exceeded physiological values [32]. Further reductions were not possible within the technical restrictions of this study. As the pressure sensors were positioned upstream of the flow-through model (Fig. 1), the pressures inside the model can be presumed to be smaller than those measured by the sensors [43]. Additionally, unlike the case shown in Fig. 1, the erythrocyte concentrate was not fed back to the reservoir, so only fresh concentrate streamed through the glass pulp model each time. This avoided multiple exposure of the blood cells to high pressures. Measurements with a constant blood flow could not be performed with the glass pulp model because the static pressure generated by the burettes was too small to create sufficient erythrocyte flow due to the high flow resistance of the narrow tubes and the glass pulp.

In summary, we tried to take into account as many factors as possible for the design of the glass pulp model simulating the in vivo situation. Nevertheless, the system has methodological limitations, especially concerning the simulation of (micro) vascular circulation, the optical properties of a human jaw and tooth or the simulation of possible movement artefacts in clinical situations. 
Despite the reduction in volumetric blood flow and the reduction of the emulated tissue volumes to in vivo scales, it was possible to record synchronously pulse modulated voltage signals (PPG signals) from pulsed blood flow and to determine $\Delta \mathrm{U}$ values for $\mathrm{T}, \mathrm{G}$ and $\mathrm{TG}$ at $625 \mathrm{~nm}$ and for T and TG at $940 \mathrm{~nm}$.

While gingival signals $(\mathrm{G})$ exceeded pulpal signals $(\mathrm{T})$ at $940 \mathrm{~nm}$ in measurements with the tooth-gingiva-model (TGM) from Niklas et al. [17], with the glass pulp model (GPM) gingival signals $(\mathrm{G})$ were not detectable at $940 \mathrm{~nm}$. This can probably be explained by the different designs of the two models and underpins the necessity to perform such in vitro experiments in models which emulate in vivo conditions as closely as possible.

The in vitro detection of gingival signals in measurements on the tooth at $625 \mathrm{~nm}$ shows that rays from LEDs which are directed onto the dental crown can be scattered via the dental hard tissues into surrounding structures. This is in line with in vivo studies with the PPG and PO methods, which demonstrated the influence of circulation in other than pulpal tissues on signals detected through teeth [6, 18-20]. The fact that the gingival erythrocyte flow could not be detected at $940 \mathrm{~nm}$, does not exclude the influence of non-pulpal signals on PPG measurements at this wavelength in vivo, as first reports on in vivo PPG and PO signals from non-vital teeth indicate $[6,19,20]$. It must be emphasized that only the blood flow of the marginal gingiva was emulated in this study. In vivo, blood flow in other structures, such as the periodontium [18, 30, 38], lip and tongue [6, 44] pose additional sources for non-pulpal PPG signals, as has already been shown for LDF signals [22, 23, 45].

The $\Delta U$ values of $T$ and TG were smaller at $625 \mathrm{~nm}$ than at $940 \mathrm{~nm}$. This wavelength dependency may arise from the optical properties of both dental and surrounding tissues. Hirmer et al. [46] applying infrared and terahertz spectroscopy [47-49] in a wide spectral range from visible to far infrared wavelengths showed that from $600 \mathrm{~nm}$ to $1400 \mathrm{~nm}$, teeth demonstrate a high degree of transmission. Within this "transmission window" [46], at $625 \mathrm{~nm}$ transmission is lower than at $940 \mathrm{~nm}$ which matches the results from other studies 
$[50,51]$. On the other hand, the glass pulp may also influence the transmission properties of the model, even though with the tooth gingiva model $\Delta U$ values at $625 \mathrm{~nm}$ were also smaller than those at $940 \mathrm{~nm}$.

The results for the erythrocyte concentrate from donor 3 (EC 3) showed a lower voltage signal amplitude than for the one from donor 4 (EC 4), as the optical properties of blood differ between individuals $[52,53]$. Nevertheless, the ranking of pulpal $(T)$, gingival $(G)$ and combined blood flow (TG) were consistent between both donors and at both wavelengths.

The experiments with the glass pulp model and pump cessation during signal recording showed similar results to those with the tooth gingiva model. These results also illustrate the insensitivity of the signal detection system towards static blood and extrinsic signal sources with the new glass pulp model.

Photoplethysmography realized in this paper appears to be suitable for detecting pulpal blood flow in teeth and thus to be a clinically valuable method for evaluating pulp vitality. Signals from blood flow in other oral tissues like the gingiva could be reduced by selecting a suitable infrared wavelength.

Taking the results of this research to their logical conclusion, there is much room for miniaturization. The apparatus shown in figure 1 was not intended as a final product and much development would be required before marketable equipment emerges. Nevertheless, some thoughts in this direction have been made. The "lab on a chip" concept is now well established. In fact, the Royal Society of Chemistry launched a journal with the title "Lab on a chip" in 2001.

Although "chip" usually implies an electronic device, most such systems rely on capillary transport of fluids and evaluation through sensors (both integrated and discrete) including electro-optical signal detection [54] similar to that discussed here. Realization of Photoplethysmography apparatus in such a form is quite conceivable. This was not followed 
during this work as the focus remained on basic research and proof of concept. Future developments could easily change this.

\section{Conclusions}

The new PPG signal recording system proved to be suitable for the detection of pulsed blood flow in our experimental design, providing larger signal amplitudes than in previous systems whilst remaining insensitive to constant, non-pulsed or no blood flow.

Using this new recording system, pulpal blood signals could be detected in vitro at 625 and $940 \mathrm{~nm}$ in a model resembling in vivo conditions in size and volumetric blood flow rates. Interference of signals from the gingiva in this model was apparent only at $625 \mathrm{~nm}$, not at 940 nm.

The PPG-based recording of pulsed blood signals presents a promising technique for future pulp vitality testing, though a clear differentiation between pulpal and non-pulpal PPG signals is crucial.

\section{Compliance with Ethical Standards}

Conflict of Interest: All authors declare that they have no conflict of interest.

Funding: The work was supported by the DFG projects (SCHM 386/3, GA-501/10 and MO 2196/1), the Linkage Grant of IB of BMBF at DLR and OTH-Regensburg Applications Center “Miniaturisierte Sensorik” (SappZ) funded by the Bavarian Government.

Ethical approval: This article does not contain any studies with human participants or animals performed by any of the authors.

Informed consent: For this type of study, formal consent is not required. 


\section{References}

1. Levin LG (2013) Pulp and Periradicular Testing. J Endod 39:S13-S19. doi: 10.1016/j.joen.2012.11.047

2. Mejàre IA, Axelsson S, Davidson T, et al (2012) Diagnosis of the condition of the dental pulp: a systematic review. Int Endod J 45:597-613. doi: 10.1111/j.1365-2591.2012.02016.x

3. Chen E, Abbott PV (2009) Dental pulp testing: a review. Int J Dent 2009:365785-12. doi: $10.1155 / 2009 / 365785$

4. Yu C, Abbott PV (2007) An overview of the dental pulp: its functions and responses to injury. Aust Dent J 52:S4-S6. doi: 10.1111/j.1834-7819.2007.tb00525.x

5. Allen J (2007) Photoplethysmography and its application in clinical physiological measurement. Physiol Meas 28:R1-R39. doi: 10.1088/0967-3334/28/3/R01

6. Fein ME, Gluskin AH, Goon WW, et al (1997) Evaluation of optical methods of detecting dental pulp vitality. J Biomed Opt 2:58-73. doi: 10.1117/12.261679

7. Oikarinen KS, Kainulainen V, Särkelä V, et al (1997) Information of circulation from soft tissue and dental pulp by means of pulsatile reflected light: further development of optical pulp vitalometry. Oral Surg Oral Med Oral Pathol Oral Radiol Endod 84:315-320.

8. Carlson KA, Jahr JS (1993) A historical overview and update on pulse oximetry. Anesthesiol Rev 20:173-181.

9. Jafarzadeh H, Rosenberg PA (2009) Pulse Oximetry: Review of a Potential Aid in Endodontic Diagnosis. J Endod 35:329-333. doi: 10.1016/j.joen.2008.12.006

10. Schnettler JM, Wallace JA (1991) Pulse oximetry as a diagnostic tool of pulpal vitality. J Endod 17:488-490. doi: 10.1016/S0099-2399(06)81795-4

11. Jafarzadeh H (2009) Laser Doppler flowmetry in endodontics: a review. Int Endod J 42:476490. doi: 10.1111/j.1365-2591.2009.01548.x

12. Kimura Y, Wilder-Smith P, Matsumoto K (2000) Lasers in endodontics: a review. Int Endod J 33:173-185.

13. Dick SK, Chistyakova GG, Terekh AS, et al (2014) Characterization of blood flow rate in dental pulp by speckle patterns of backscattered light from an in vivo tooth. J Biomed Opt 19:106012. doi: 10.1117/1.JBO.19.10.106012

14. Stoianovici C, Wilder-Smith P, Choi B (2011) Assessment of pulpal vitality using laser speckle imaging. Lasers Surg Med 43:833-837. doi: 10.1002/lsm.21090

15. Yoon M-J, Kim E, Lee S-J, et al (2010) Pulpal Blood Flow Measurement with Ultrasound Doppler Imaging. J Endod 36:419-422. doi: 10.1016/j.joen.2009.12.031

16. Cho Y-W, Park S-H (2014) Use of ultrasound Doppler to determine tooth vitality in a discolored tooth after traumatic injury: its prospects and limitations. Restor Dent Endod 39:6873. doi: 10.5395/rde.2014.39.1.68

17. Niklas A, Hiller KA, Jaeger A, et al (2014) In vitro optical detection of simulated blood pulse in a human tooth pulp model. Clinical Oral Investigations 18:1401-1409. doi: 10.1007/s00784- 
013-1115-z

18. Miwa Z, Ikawa M, Iijima $\mathrm{H}$, et al (2002) Pulpal blood flow in vital and nonvital young permanent teeth measured by transmitted-light photoplethysmography: a pilot study. Pediatr Dent 24:594-598.

19. Karayilmaz H, Kirzioğlu Z (2011) Comparison of the reliability of laser Doppler flowmetry, pulse oximetry and electric pulp tester in assessing the pulp vitality of human teeth. J Oral Rehabil 38:340-347. doi: 10.1111/j.1365-2842.2010.02160.x

20. Siddheswaran V, Adyanthaya R, Shivanna V (2011) Pulse oximetry: a diagnostic instrument in pulpal vitality testing - an in vivo study. World Journal of Dentistry 2:225-230.

21. Ingólfsson AR, Tronstad L, Hersh EV, Riva CE (1994) Efficacy of laser Doppler flowmetry in determining pulp vitality of human teeth. Endod Dent Traumatol 10:83-87.

22. Polat S, Er K, Akpinar KE, Polat NT (2004) The sources of laser Doppler blood-flow signals recorded from vital and root canal treated teeth. Arch Oral Biol 49:53-57. doi: 10.1016/S00039969(03)00197-3

23. Soo-ampon S, Vongsavan N, Soo-ampon M, et al (2003) The sources of laser Doppler bloodflow signals recorded from human teeth. Arch Oral Biol 48:353-360. doi: 10.1016/S00039969(03)00011-6

24. Akpinar KE, Er K, Polat S, Polat NT (2004) Effect of gingiva on laser Doppler pulpal blood flow measurements. J Endod 30:138-140. doi: 10.1097/00004770-200403000-00003

25. Hartmann A, Azérad J, Boucher Y (1996) Environmental effects on laser Doppler pulpal blood-flow measurements in man. Arch Oral Biol 41:333-339. doi: 10.1016/00039969(95)00133-6

26. Roebuck EM, Evans DJP, Stirrups D, Strang R (2001) The effect of wavelength, bandwidth, and probe design and position on assessing the vitality of anterior teeth with laser Doppler flowmetry. Int J Pediatr Dent 10:213-220. doi: 10.1046/j.1365-263x.2000.00194.x

27. Polat S, Er K, Polat NT (2005) Penetration depth of laser Doppler flowmetry beam in teeth. Oral Surg Oral Med Oral Pathol Oral Radiol Endod 100:125-129. doi: 10.1016/j.tripleo.2004.11.018

28. Ikawa M, Vongsavan N, Horiuchi H (1999) Scattering of laser light directed onto the labial surface of extracted human upper central incisors. J Endod 25:483-485. doi: 10.1016/S00992399(99)80286-6

29. Kakino S, Miwa Z, Kirimoto A, et al (2007) A new multi-wavelength optical-plethysmograph for quantitative determination of pulpal hemoglobin content and oxygen level using green and near-infrared LEDs. Proc SPIE 6425:642508-642508-9. doi: 10.1117/12.699628

30. Kakino S, Kushibiki S, Yamada A, Miwa Z (2013) Optical Measurement of Blood Oxygen Saturation of Dental Pulp. ISRN Biomedical Engineering 2013:1-6. doi: 10.1155/2013/502869

31. Lindberg L-G, Öberg PA (1993) Optical properties of blood in motion. Opt Eng 32:253-257. doi: $10.1117 / 12.60688$

32. Kim S (1985) Microcirculation of the dental pulp in health and disease. J Endod 11:465-471. doi: 10.1016/S0099-2399(85)80219-3 
33. Path MG, Meyer MW (1980) Heterogeneity of blood flow in the canine tooth in the dog. Arch Oral Biol 25:83-86. doi: 10.1016/0003-9969(80)90081-3

34. Hock J, Nuki K, Schlenker R, Hawks A (1980) Clearance rates of Xenon-133 in non-inflamed and inflamed gingiva of dogs. Arch Oral Biol 25:445-449. doi: 10.1016/0003-9969(80)900503

35. Schulz I, Putzger J, Niklas A, et al PPG signal acquisition and analysis on in vitro tooth model for dental pulp vitality assessment. ARC 2012. Submission 16

36. Hiller K-A, Christa T, Niklas A, et al (2013) An in-vitro-model of a human jaw for testing optical properties. J Dent Res 92:

37. Diaz-Arnold AM, Wilcox LR, Arnold MA (1994) Optical detection of pulpal blood. J Endod 20:164-168. doi: 10.1016/S0099-2399(06)80327-4

38. Kahan RS, Gulabivala K, Snook M, Setchell DJ (1996) Evaluation of a pulse oximeter and customized probe for pulp vitality testing. J Endod 22:105-109. doi: 10.1016/S00992399(96)80283-4

39. Friebel M, Roggan A, Müller G, Meinke M (2006) Determination of optical properties of human blood in the spectral range 250 to $1100 \mathrm{~nm}$ using Monte Carlo simulations with hematocrit-dependent effective scattering phase functions. J Biomed Opt 11:034021. doi: $10.1117 / 1.2203659$

40. Vongsavan N, Matthews B (1993) Experiments on extracted teeth into the validity of using laser Doppler techniques for recording pulpal blood flow. Arch Oral Biol 38:431-439. doi: 10.1016/0003-9969(93)90215-8

41. Margolis J (1957) Initiation of blood coagulation by glass and related surfaces. J Physiol 137:95-109.

42. Ketterl W (1983) Age-induced changes in the teeth and their attachment apparatus. Int Dent J 33:262-271.

43. Benedict RP (1984) Fundamentals of Temperature, Pressure and Flow Measurements. John Wiley \& Sons

44. Oikarinen K, Kopola H, Mäkiniemi M, Herrala E (1996) Detection of pulse in oral mucosa and dental pulp by means of optical reflection method. Endod Dent Traumatol 12:54-9.

45. Hoke JA, Burkes EJ, White JT, et al (1994) Blood-flow mapping of oral tissues by laser Doppler flowmetry. Int J Oral Maxillofac Surg 23:312-315. doi: 10.1016/S09015027(05)80117-1

46. Hirmer M, Danilov SN, Giglberger S, et al (2012) Spectroscopic study of human teeth and blood from visible to terahertz frequencies for clinical diagnosis of dental pulp vitality. J Infrared Milli Terahz Waves 33:366-375. doi: 10.1007/s10762-012-9872-3

47. Ganichev S, Prettl W (2006) Intense Terahertz Excitation of Semiconductors. Oxford University Press on Demand

48. Olbrich P, Karch J, Ivchenko EL, et al (2011) Classical ratchet effects in heterostructures with a lateral periodic potential. Phys Rev B 83:165320. doi: 10.1103/PhysRevB.83.165320 
49. Lechner V, Golub LE, Olbrich P, et al (2009) Tuning of structure inversion asymmetry by the $\delta$-doping position in (001)-grown GaAs quantum wells. Appl Phys Lett 94:242109. doi: $10.1063 / 1.3156027$

50. Alfano R, Lam W, Zarrabi H, et al (1984) Human teeth with and without caries studied by laser scattering, fluorescence, and absorption spectroscopy. IEEE J Quantum Electron 20:15121516. doi: 10.1109/JQE.1984.1072351

51. Ikawa M, Horiuchi H, Ikawa K (1994) Optical characteristics of human extracted teeth and the possible application of photoplethysmography to the human pulp. Arch Oral Biol 39:821-827. doi: 10.1016/0003-9969(94)90012-4

52. Hammer M, Schweitzer D, Michel B, et al (1998) Single scattering by red blood cells. Appl Opt 37:7410-7418.

53. Meinke M, Müller G, Helfmann J, Friebel M (2007) Empirical model functions to calculate hematocrit-dependent optical properties of human blood. Appl Opt 46:1742-1753.

54. Huerre A, Jullien MC, Theodoly O, Valignat MP (2016) Absolute 3D reconstruction of thin films topography in microfluidic channels by interference reflection microscopy. Lab Chip 16:911-916. doi: 10.1039/C5LC01417D 
Fig. 1 Experimental configuration for (a) pulsed circulation and (b) constant, non-pulsed circulation (flow direction indicated by arrows): (1) peristaltic pumps, (2) erythrocyte concentrate reservoir, (3) light source, (4) flow through model, (5) signal detecting components, (6) pressure sensors, (7) burettes

Fig. 2 Schematic drawing of the glass pulp model with (1) tooth, (2) glass pulp, (3) solid resin jaw, (4) soft silicone gingiva and (5) silicone tube

Fig. 3 Glass pulp with attached circulation tubes placed into one model tooth half

Fig. 4 Example recording of a modulating signal with illustration of the quantitative analysis method. The first three seconds of the recording were not analyzed. The amplitudes $(\Delta \mathrm{U} 1, \Delta \mathrm{U} 2$ and $\Delta \mathrm{U} 3)$ of the following three modulated signals were calculated as the difference of the respective maximum and minimum values of each of the three modulations, from which the median was calculated as the representative value for this experiment

Fig. 5 Amplitudes of pulse synchronous voltage modulation ( $\Delta \mathrm{U}[\mathrm{V}]$, medians with $25 \%$ - and $75 \%$-quantiles), measured with pulsed circulation and the tooth-gingiva-model for a separate circulation in the tooth (T) or the gingiva (G) and both combined (TG) at two wavelengths (red and infrared); all values were pairwise significantly different except the pair marked with *

Fig. 6 Example recordings for constantly, non-pulsed circulation in the tooth ( $T$, top row), the gingiva (G, middle row) and tooth and gingiva combined (TG, bottom row) for both wavelengths $625 \mathrm{~nm}$ (left column) and $940 \mathrm{~nm}$ (right column)

Fig. 7 Example recordings of the voltage signal (Voltage; yellow) and vascular pressure signals (Pressure; pulpal pressure, blue upper curve and gingival pressure, red lower curve) before and after cessation of the pulsed circulation in the tooth gingiva model at the 10th second. Samples show the flow modes tooth (T, top row), gingiva (G, middle row) and tooth and gingiva (TG, bottom row) for the two wavelengths 625 nm (left column) and $940 \mathrm{~nm}$ (right column) 
Fig. 8 Amplitudes of pulse synchronous voltage modulation signals ( $\Delta \mathrm{U}[\mathrm{V}]$, medians with $25 \%$ - and $75 \%$ quantiles), measured with pulsed circulation and the glass pulp model for a separate circulation in the tooth (T) or the gingiva (G) and both combined (TG) at two wavelengths (red and infrared) and with two different erythrocyte concentrates (EC 3 and EC 4); significance analysis was performed separately for each wavelength and EC; all values were pairwise significantly different except the pairs marked with "a“ to “d

Fig. 9 Example recordings of the voltage signal (Voltage, yellow) and vascular pressure signals (Pressure; pulpal pressure, blue upper curve and gingival pressure, red lower curve) before and after cessation of the pulsed circulation in the glass pulp model at the 10th second. Samples show the flow modes tooth (T, top row), gingiva (G, middle row) and tooth and gingiva (TG, bottom row) for the two wavelengths $625 \mathrm{~nm}$ (left column) and $940 \mathrm{~nm}$ (right column)

Fig. 10 Results taken over from Niklas et al [17], only the four red bars depicting 'no shielding'. The four red bars there depict the results four experiments consisting of five independent measurements, As these data were derived during an earlier stage of one and the same project in our laboratory, we recalculated these 20 independent samples and depict them in the format of the present presentation: Amplitudes of pulse synchronous voltage modulation signals ( $\Delta \mathrm{U}[\mathrm{V}]$, medians with $25 \%$ - and $75 \%$-quantiles), measured with pulsed circulation and the tooth gingiva model for a separate circulation in the tooth (our study: T; other study: TA) or the gingiva (our study: G; Other study: GA) and both combined (our study: TG; Other study: TAG) at two wavelengths ( red and infrared). 'Flow Mode' in the present study was 'Perfused component of TGM' in the other study, where 'TGM' stands for 'tooth-gingiva-model'.

Fig. 11 Transmission of the porcine mandible (dotted lines), Paladur pink $1.18 \mathrm{~g}$ and Paladur clear $0.12 \mathrm{~g}$ (D2), Paladur pink 1.16 g and Paladur clear 0.34 g (D3), Paladur pink 1.14 g and Paladur clear 0.36 g (D4) and Paladur pink $1.12 \mathrm{~g}$ and Paladur clear $0.38 \mathrm{~g}$ (D5). Best match compared to reference: mixture of $1.14 \mathrm{~g}$ pink and $0.36 \mathrm{~g}$ clear Paladur (D4) [36] 